\title{
ZEROS OF SOLUTIONS AND OF WRONSKIANS FOR THE DIFFERENTIAL EQUATION $L_{n} y+p(x) y=0$
}

\author{
URI ELIAS
}

\begin{abstract}
The equation which is studied here is $L_{n} y+p(x) y=0, a \leq x \leq b$, where $L_{n}$ is a disconjugate differential operator and $p(x)$ is of a fixed sign. We prove that certain solutions of the equation and corresponding odd-order minors of the Wronskian have an equal number of zeros, and we apply this property to oscillation problems.
\end{abstract}

\section{INTRODUCTION}

In a recent work [4], Etgen, Jones, and Taylor investigated ways of factoring the differential equation

$$
L_{n} y+p(x) y=0, \quad a<x<b,
$$

where $p(x)$ is continuous and of one sign and $L_{n}$ is disconjugate, into a product of lower order operators. Their investigation was based on relations between the nonvanishing of certain minors of the Wronskian and disconjugacy properties of equation (1.1). In this paper we extend the results of [4] and study, more generally, how many zeros certain minors of the Wronskian have. We shall show that certain odd order minors of the Wronskian and related solutions of (1.1) have the same number of zeros. This property will be interpreted in terms of the oscillation theory of (1.1).

Let the disconjugate differential operator $L_{n}$ be

$$
L_{n} y=\rho_{n}\left(\rho_{n-1} \cdots\left(\rho_{1}\left(\rho_{0} y\right)^{\prime}\right)^{\prime} \cdots\right)^{\prime},
$$

with weight functions $\rho_{i}>0, \rho_{i} \in C^{n-i}, i=0, \ldots, n$. We denote $L_{0} y=\rho_{0} y$ and $L_{i} y=\rho_{i}\left(L_{i-1} y\right)^{\prime}, i=1, \ldots, n$, and call $L_{0} y, \ldots, L_{n} y$ the quasiderivatives of $y$.

Our results will be formulated in terms of a special basis $\left\{u_{0}(x), \ldots, u_{n-1}(x)\right\}$ of the solution space of (1.1), which has been used in [1-5]. Since $u_{0}(x), \ldots$, $u_{n-1}(x)$ will be defined in the next section by boundary value problems $(2.2)_{0}-$ $(2.2)_{n-1}$, we first mention a known property [7] of boundary value problems of

Received by the editors May 17, 1988 .

1980 Mathematics Subject Classification (1985 Revision). Primary 34C10, 34A30.

Key words and phrases. Linear differential equations, Wronskian, zeros of solutions.

This research was supported by the Fund for the Promotion of Research at the Technion. 
equation (1.1): If $k$ quasiderivatives of a solution $y$ vanish at the endpoint $a$ and $n-k$ quasiderivatives of $y$ vanish at $b, a<b$, then $n-k$ must be odd if $p(x)>0$ and even if $p(x)<0$. Therefore we shall say that an integer $k$, $0 \leq k \leq n$, is of admissible parity if

$$
(-1)^{n-k} p(x)<0 \text {. }
$$

In [4], the authors studied the nonvanishing of certain minors of odd order of the Wronskian. Theorems 7, 8 and 13,14 of [4] motivate our work. For example,

Theorem [4, Theorems 13, 14]. Let $l<k$ be of admissible parity and $u_{l-1}, \ldots$, $u_{k-2}, u_{k-1}$ elements of the basis which is defined by (2.2). If equation (1.1) is $(k, n-k)$-disconjugate on $[a, b]$, then

$$
W\left(u_{l-1}, \ldots, u_{k-2}, u_{k-1}\right) \neq 0, \quad a<x<b .
$$

Conversely, if (1.1) is not $(k, n-k)$-disconjugate on $[a, b]$ and $b$ is not a $(k, n-k)$-type conjugate point of $x=a$, then $W\left(u_{l-1}, \ldots, u_{k-2}, u_{k-1}\right)$ must have a zero on $(a, b)$.

Our aim is to generalize this result:

Theorem 1.1. Let $l<k, q$ be of admissible parity. If $q \geq k$ then for arbitrary constants $\alpha, \beta$, not both zero, the solution $\alpha u_{q-1}+\beta u_{q}$ and the minor $W\left(u_{l-1}, \ldots, u_{k-2}, \alpha u_{q-1}+\beta u_{q}\right)$ have an equal number of zeros, all of them simple, in $(a, b)$. If $q \leq l-2$, then the numbers of the above-mentioned zeros differ at most by 1 .

Theorem 1.1 has a simple interpretation in view of the results of [2]. In [2] we proved that for every solution $y$ of (1.1), the function

$$
z=W\left(u_{l-1}, \ldots, u_{k-2}, y\right)
$$

is a solution of an equation

$$
M_{n} z+p(x) z=0, \quad a<x<b,
$$

where $M_{n} z=\sigma_{n}\left(\sigma_{n-1} \cdots\left(\sigma_{1}\left(\sigma_{0} z\right)^{\prime}\right)^{\prime} \cdots\right)^{\prime}, \sigma_{0}, \ldots, \sigma_{n}$ depend only on $u_{l-1}$, $\ldots, u_{k-2}$, and

$$
\sigma_{0} \cdots \sigma_{n}=\rho_{0} \cdots \rho_{n} .
$$

We called $\left\{u_{l-1}, \ldots, u_{k-2}\right\}$ a pivotal block and equation (1.3) the equation associated with equation (1.1) and this pivotal block. Let us denote

$$
z_{i}=W\left(u_{l-1}, \ldots, u_{k-2}, u_{i}\right), \quad i \notin\{l-1, \ldots, k-2\} .
$$

In these terms Theorem 1.1 may be stated as follows:

Theorem 1.2. For arbitrary constants $\alpha, \beta$ the solution $\alpha u_{q-1}+\beta u_{q}$ of (1.1) and the solution $\alpha z_{q-1}+\beta z_{q}=W\left(u_{l-1}, \ldots, u_{k-2}, \alpha u_{q-1}+\beta u_{q}\right)$ of the associated equation (1.3) have an equal number of zeros in $(a, b)$ if $q \geq k$, and the number of these zeros differ at most by 1 if $q \leq l-2$. 


\section{Definitions}

Before we begin the actual proof, we list some definitions and results from $[1,2]$. Let $S\left(c_{0}, \ldots, c_{n}\right)$ denote the number of sign changes in the sequence $c_{0}, \ldots, c_{n}$ of nonzero numbers. For a solution $y$ of $(1.1)$ we define

$$
\begin{aligned}
& S\left(y, x^{+}\right)=\lim _{\xi \rightarrow x^{+}} S\left(L_{0} y(\xi),-L_{1} y(\xi), \ldots,(-1)^{n} L_{n} y(\xi)\right), \\
& S\left(y, x^{-}\right)=\lim _{\xi \rightarrow x^{-}} S\left(L_{0} y(\xi), L_{1} y(\xi), \ldots, L_{n} y(\xi)\right) .
\end{aligned}
$$

The quantities $S\left(y, x^{+}\right), S\left(y, x^{-}\right)$are closely related to boundary value problems of (1.1). For example, if $y$ has a zero of multiplicity $q$ at $x_{0}$, then obviously

$$
S\left(y, x_{0}^{+}\right) \geq q, \quad S\left(y, x_{0}^{-}\right) \geq q .
$$

We summarize some of their useful properties:

Proposition 2.1 [1]. (a) Let $x_{1}, \ldots, x_{r}$ be the zeros of the quasiderivatives $L_{0} y$, $\ldots, L_{n-1} y$ in $(a, b)$, let $n\left(x_{i}\right)$ be the multiplicity of the zero at $x_{i}$, and let $\langle q\rangle$ be the greatest even integer not exceeding $q$. Then

$$
S\left(y, a^{+}\right)+\sum_{a<x_{i}<b}\left\langle n\left(x_{i}\right)\right\rangle+S\left(y, b^{-}\right) \leq n .
$$

(b) $S\left(y, x^{+}\right)$is an integer valued, nondecreasing function of $x$.

(c) $S\left(y, x^{+}\right)$and $n-S\left(y, x^{-}\right)$are integers of admissible parities.

Now we define a basis for the solution space of (1.1) by means of $n$ boundary value problems. For every admissible $k$ we define $u_{k-1}(x)$ as the unique solution of (1.1) which satisfies the system of boundary value conditions

$$
\begin{array}{rlrl}
L_{t} u(a) & =0, & t & =0, \ldots, k-2, k, \\
L_{k-1} u(a) & =1, & \\
L_{t} u(b) & =0, & t & =0, \ldots, n-k-2,
\end{array}
$$

and $u_{k}(x)$ as the unique solution which satisfies

$$
\begin{aligned}
L_{t} u(a) & =0, \quad t=0, \ldots, k-1, \\
L_{k} u(a) & =1, \\
L_{t} u(b) & =0, \quad t=0, \ldots, n-k-2 .
\end{aligned}
$$

Here and throughout the paper the following convention will be used: Whenever an admissible $k$ and solutions $u_{k-1}, u_{k}$ are mentioned, omit $u_{-1}($ if $k=0)$ or $u_{n}($ if $k=n)$ which are not defined at all.

It is proved in [1,4] that the set $\left\{u_{0}, \ldots, u_{n-1}\right\}$ which is defined in this way, exists, it is unique, and it is a basis of solutions of (1.1). This basis had been used extensively in [1-5].

Let the Wronskian be

$$
W\left(u_{0}, \ldots, u_{n-1}\right)=\operatorname{det}\left(L_{i} u_{j}\right)_{i, j=0, \ldots, n-1}
$$


and let its minors be denoted by

$$
W\left(\begin{array}{c}
u_{i_{1}}, \ldots, u_{i_{r}} \\
j_{1}, \ldots, j_{r}
\end{array}\right)=\operatorname{det}\left(L_{j_{s}} u_{i_{t}}(x)\right)_{s, t=1, \ldots, r} .
$$

The properties of the basis $u_{0}, \ldots, u_{n-1}$ which will be needed are the following: Proposition $2.2[1,4]$. Let $u_{0}, \ldots, u_{n-1}$ be the basis of solutions as defined by $(2.2)_{0}-(2.2)_{n-1}$. If $r \leq q$ are of admissible parities, then for arbitrary constants $c_{r-1}, \ldots, c_{q}$

$$
\begin{gathered}
r \leq S\left(c_{r-1} u_{r-1}+\cdots+c_{q} u_{q}, x^{+}\right) \leq q \quad \text { on }[a, b) \\
W\left(\begin{array}{c}
u_{r-1}, \ldots, u_{q} \\
i, \ldots, i+q-r+1
\end{array}\right) \neq 0, \quad i=0, \ldots, n-q+r-2, \text { on }(a, b)
\end{gathered}
$$

(recall the convention about $u_{-1}, u_{n}$ !).

Most frequently we shall use that for admissible $l<k$,

$$
W\left(u_{l-1}, u_{l}, \ldots, u_{k-1}, u_{k}\right) \neq 0, \quad a<x<b .
$$

The results of [2] about the associated equation are the following:

Proposition 2.3 [2]. Let $l \leq k$ be two integers of admissible parity. For every solution $y$ of (1.1), the function

$$
z=W\left(u_{l-1}, \ldots, u_{k}, y\right)
$$

is a solution of the differential equation

$$
M_{n} z+p(x) z=0, \quad a<x<b,
$$

where $M_{0} z=\sigma_{0} z, M_{i} z=\sigma_{i}\left(M_{i-1} z\right)^{\prime}, \sigma_{i}>0$ on $(a, b), i=0, \ldots, n$, and $\sigma_{0}(x), \ldots, \sigma_{n}(x)$ depend only on $u_{l-1}, \ldots, u_{k}$ and satisfy

$$
\sigma_{0} \cdots \sigma_{n}=\rho_{0} \cdots \rho_{n} \text {. }
$$

If $r \leq q$ are of admissible parity such that $\{l-1, \ldots, k\},\{r-1, \ldots, q\}$ are disjoint, then the solutions

$$
z_{j}=W\left(u_{l-1}, \ldots, u_{k}, u_{j}\right), \quad j=r-1, \ldots, q,
$$

of (2.6) satisfy for arbitrary constants $c_{r-1}, \ldots, c_{q}$

$$
W_{M}\left(\begin{array}{c}
z_{r-1}, \ldots, z_{q} \\
i, \ldots, i+q-r+1
\end{array}\right) \neq 0, \quad i=0, \ldots, n-q+r-2, \text { on }(a, b),
$$

(Here and elsewhere the subscripts $M, L$ will remind us that the quasiderivatives in question are $M_{i} z, L_{i} u$ respectively.)

It should be emphasized that (2.6) is singular at $x=a$ and $x=b$. The quasiderivatives $M_{i} z$ are given by

$$
M_{i} z=W\left(\begin{array}{c}
u_{l-1}, u_{l}, \ldots, u_{k}, y \\
i, i+1, \ldots, i+k-l+2
\end{array}\right) / W\left(\begin{array}{c}
u_{l-1}, \ldots, u_{k} \\
i+1, \ldots, i+k-l+2
\end{array}\right),
$$


where $i=0, \ldots, n-k+1-3$ and $a<x<b$. When $i+k-1+2 \geq n$, the explicit expression for $M_{i} z$ is slightly different (see [2]). Since we do not need it here, it is omitted.

\section{Proof of Theorem 1.1 For $q=k$}

The proof of Theorem 1.1 is divided into several short propositions.

Proposition 3.1. $\alpha u_{q-1}+\beta u_{q}$ and $\alpha z_{q-1}+\beta z_{q}$ have no multiple zeros in $(a, b)$.

Indeed, if we take $r=q$ in (2.3) and (2.6), we get

$$
S_{L}\left(\alpha u_{q-1}+\beta u_{q}, x^{+}\right) \equiv S_{M}\left(\alpha z_{q-1}+\beta z_{q}, x^{+}\right) \equiv q, \quad a<x<b .
$$

Consequently,

$$
\begin{gathered}
S\left(\alpha u_{q-1}+\beta u_{q}, a^{+}\right)=q, \\
S\left(\alpha u_{q-1}+\beta u_{q}, b^{-}\right)=S\left(\alpha u_{q-1}+\beta u_{q},(b-\varepsilon)^{-}\right) \\
=n-S\left(\alpha u_{q-1}+\beta u_{q},(b-\varepsilon)^{+}\right)=n-q
\end{gathered}
$$

and, by (2.1), the solution $\alpha u_{q-1}+\beta u_{q}$ of (1.1) may have no multiple zeros in $(a, b)$. The same argument shows that the solution $\alpha z_{q-1}+\beta z_{q}$ of (1.3) also has no multiple zeros in $(a, b)$.

From here on we separate the cases $q=k$ and $q \neq k$. In this section we prove the theorem for $q=k$ only, while the case $q>k$ will be discussed in the following section. The reason for this separation will be clarified soon.

Let $x_{0}$ be a zero of $\alpha z_{k-1}+\beta z_{k}$, that is

$$
W\left(u_{l-1}, \ldots, u_{k-2}, \alpha u_{k-1}+\beta u_{k}\right)\left(x_{0}\right)=0 .
$$

Then there exists a combination $c_{l-1} u_{l-1}+\cdots+c_{k-2} u_{k-2}+c_{k-1}\left(\alpha u_{k-1}+\beta u_{k}\right)$ which has a zero of multiplicity $k-l+1$ at $x_{0}$. Now, for every $s \in(a, b)$, we take a combination of $u_{l-1}, \ldots, u_{k-2}, \alpha u_{k-1}+\beta u_{k}$ which has at $x=s$ a zero of multiplicity at least $k-l$. Such a combination obviously exists and as $W\left(u_{l-1}, \ldots, u_{k-2}\right)(s) \neq 0$ for all $a<s<b$, the combination is essentially unique and may be written, up to a constant

$$
u(x, s)=\left|\begin{array}{cccc}
u_{l-1}(x), & \ldots, & u_{k-2}(x), & \alpha u_{k-1}+\beta u_{k}(x) \\
L_{0} u_{l-1}(s), & \ldots, & L_{0} u_{k-2}(s), & L_{0}\left(\alpha u_{k-1}+\beta u_{k}\right)(s) \\
\vdots & & & \vdots \\
L_{k-l-1} u_{l-1}(s), & \ldots, & & L_{k-l-1}\left(\alpha u_{k-1}+\beta u_{k}\right)(s)
\end{array}\right| .
$$

More explicitly,

$$
u(x, s)=c_{l-1}(s) u_{l-1}+\cdots+c_{k-2}(s) u_{k-2}+c_{k-1}(s)\left(\alpha u_{k-1}+\beta u_{k}\right)
$$

and

$$
c_{k-1}(s)=W\left(u_{l-1}, \ldots, u_{k-2}\right)(s) \neq 0 .
$$

By (2.3), $u(x, s)$ satisfies $S\left(u, a^{+}\right) \geq l, S\left(u, b^{-}\right) \geq n-k$. At $x=s, u(x, s)$ 
has a zero of multiplicity $k-l$ and $k-l$ is even, therefore $S\left(u, a^{+}\right)+\langle n(s)\rangle+$ $S\left(u, b^{-}\right) \geq l+(k-l)+(n-k)=n$. It follows by $(2.1)$ that $u(x, s)$ and its quasiderivatives have no multiple zeros in $(a, s) \cup(s, b)$. (If $\alpha u_{k-1}+\beta u_{k}$ is replaced in (3.2) by $\alpha u_{q-1}+\beta u_{q}, q>k$, the last conclusion is definitely false. This is why we separate the cases $q=k$ and $q>k$ ). By the same reason it follows also from (2.1) that the multiplicity of the zero at $x=s$ can be either $k-l$ or $k-l+1$ but not more. Now,

$$
\left.L_{k-l} u(x, s)\right|_{x=s}=W\left(u_{l-1}, \ldots, u_{k-2}, \alpha u_{k-1}+\beta u_{k}\right)(s) .
$$

Therefore, the multiplicity of the zero of $u(x, s)$ at $s$ is exactly $k-l+1$ if and only if $s$ is a (simple) zero of $\alpha z_{k-1}+\beta z_{k}$. For every other $s \in(a, b)$, $u(x, s)$ has at $x=s$ a zero of exact multiplicity $k-l$. This characterization will be used to count the zeros of $\alpha z_{k-1}+\beta z_{k}$ by tracing the zeros of $u(x, s)$. More exactly, in the next propositions the following will be proved: $u(x, s)$ has for $s \rightarrow a^{+}$as many simple zeros in $(s, b)$ as $\alpha u_{k-1}+\beta u_{k}$ has in $(a, b)$; as $s$ increases, zeros of $u(x, s)$ leave $(s, b)$ one by one whenever $s$ crosses a zero of $\alpha z_{k-1}+\beta z_{k}$; when $s \rightarrow b^{-}, u(x, s)$ has no zeros in $(s, b)$.

Proposition 3.2. One simple zero of $u(x, s)$ passes from $(s, b)$ to $(a, s)$ when and only when $s$ crosses a zero of $\alpha z_{k-1}+\beta z_{k}$ in $(a, b)$ from left to right. This is the only way by which the number of the zeros of $u(x, s)$ in $(s, b)$ may change as $s$ varies in $(a, b)$.

Let $\left(\alpha z_{k-1}+\beta z_{k}\right)\left(s_{0}\right)=0$. Then $u\left(x, s_{0}\right)$ has at $x=s_{0}$ a zero of exact multiplicity $k-l+1$, while for $s$ close to $s_{0}, s \neq s_{0}, u(x, s)$ has at $x=s$ a zero of exact multiplicity $k-l$. By continuity arguments, when $s$ is sufficiently close to $s_{0}, u(x, s)$ has a unique simple zero, $\zeta(s)$, near $s_{0}$, such that $\zeta\left(s_{0}\right)=$ $s_{0}$. By the same arguments $L_{k-l} u(x, s)$ has also a unique, simple zero, say $\bar{\zeta}(s)$, near $s_{0}$, and $\bar{\zeta}\left(s_{0}\right)=s_{0}$. If we apply Rolle's theorem successively to $L_{0}(x, s), \ldots, L_{k-l}(x, s)$, we see that $\bar{\zeta}(s)$ has to be located between $\zeta(s)$ and $s$.

We want to show that $\zeta(s)<s$ when $s>s_{0}$ and $\zeta(s)>s$ when $s<s_{0}$. Since $\bar{\zeta}(s)$ is between $\zeta(s)$ and $s$, it suffices to show that $s-\bar{\zeta}(s)$ increases near $s_{0}$. Recall that $\bar{\zeta}(s)$ is the simple zero of $L_{k-l} u(x, s)$ which satisfies $\bar{\zeta}\left(s_{0}\right)=s_{0}$. By the implicit function theorem

$$
\left.\frac{d \bar{\zeta}}{d s}\right|_{s_{0}}=-\frac{\partial}{\partial s} L_{k-l} u(x, s) /\left.\frac{\partial}{\partial x} L_{k-l} u(x, s)\right|_{x=s=s_{0}} .
$$

It follows from (3.2) that $\left.(\partial / \partial s) L_{k-l}(x, s)\right|_{x=s}=0$. The denominator

$$
\left.\frac{\partial}{\partial x} L_{k-l} u(x, s)\right|_{x=s=s_{0}}=\rho_{k-l+1}^{-1}\left(s_{0}\right) L_{k-l+1} u\left(s_{0}, s_{0}\right)
$$

is not zero, otherwise $u\left(x, s_{0}\right)$ would have at $x=s_{0}$ a zero of multiplicity $k-l+2$, which we already saw to be impossible. Therefore $\bar{\zeta}^{\prime}\left(s_{0}\right)=0$, $(d / d s)(s-\bar{\zeta}(s))\left(s_{0}\right)=1$, and the first part of the proposition is proved. 
The number of the zeros of $u(x, s)$ in $(s, b)$ may change when $s$ varies in $(a, b)$ only as described above. Indeed, two zeros cannot merge at a point of $(s, b)$ since $u(x, s)$ has no multiple zeros in $(s, b)$. Neither can a zero of $u(x, s)$ tend to $b$ as $s$ varies in $(a, b)$. To show this, observe that by the boundary conditions $(2.2), u(x, s)$ satisfies

$$
\begin{gathered}
L_{t} u(b)=0, \quad t=0, \ldots, n-k-2, \\
L_{n-k-1} u(b)=c_{k-1}(s)\left(\alpha L_{n-k-1} u_{k-1}+\beta L_{n-k-1} u_{k}\right)(b), \\
L_{n-k} u(b)=c_{k-1}(s)\left(\alpha L_{n-k} u_{k-1}+\beta L_{n-k} u_{k}\right)(b),
\end{gathered}
$$

and $c_{k-1}(s) \neq 0$ for $a<s<b$. Thus, the multiplicity of the zero of $u(x, s)$ at $b$ (which is either $n-k-1$ or $n-k$ ) is equal to that of $\alpha u_{k-1}+\beta u_{k}$ and therefore is independent of $s$. Consequently, no additional zero of $u(x, s)$ meets $b$ as $s$ varies in $(a, b)$.

Next we have to discuss the situation as $s \rightarrow a^{+}$and $s \rightarrow b^{-}$.

Proposition 3.3. When $s$ is sufficiently close to $a, u(x, s)$ has in $(s, b)$ exactly as many zeros as $\alpha u_{k-1}+\beta u_{k}$ has in $(a, b)$ for all but possibly two values of $\alpha / \beta$. When $s$ is sufficiently close to $b, u(x, s)$ has no zeros in $(s, b)$ for all but possibly one value of $\alpha / \beta$.

When $s=a$ or $s=b$, the determinant (3.2) is identically zero (unless $l=0,1)$. Therefore we normalize $u(x, s)$ and define

$$
\bar{u}(x, s)=\left[c_{l-1}(s) u_{l-1}+\cdots+c_{k-2}(s) u_{k-2}+c_{k-1}(s)\left(\alpha u_{k-1}+\beta u_{k}\right)\right] / \sum_{l-1}^{k-1}\left|c_{i}(s)\right| .
$$

By the normalization, each sequence $s_{i} \rightarrow a^{+}$has a subsequence $s_{i_{j}}$ such that $u\left(x, s_{i_{j}}\right)$ converges uniformly to a nontrivial solution. $u(x, s)$ has at $x=a, s$, and $b$ zeros of multiplicities at least $l-1, k-l$, and $n-k-1$, respectively, and $\bar{u}(x, s)$ has the same ones. Hence, $\lim _{s \rightarrow a^{+}} \bar{u}(x, s)$ (or the limit of a subsequence) has at least $(l-1)+(k-l)=k-1$ zeros at $x=a$. On the other side, the limit solution is spanned by $u_{l-1}, \ldots, u_{k-2}, \alpha u_{k-1}+\beta u_{k}$, so it may have a zero of multiplicity $k-1$ at $a$ if and only if, up to a constant factor,

$$
\lim _{s \rightarrow a^{+}} \bar{u}(x, s)=\alpha u_{k-1}+\beta u_{k} .
$$

In order to prove that for $s$ sufficiently close to $a, u(x, s)$ and $\alpha u_{k-1}+\beta u_{k}$ have an equal number of zeros in $(s, b)$, it suffices to show that no zero of $u(x, s)$, except $s$, tends to the endpoints $a$ or $b$ when $s \rightarrow a^{+}$. This is definitely the case if

$$
\alpha / \beta \neq 0, \quad \alpha / \beta \neq-L_{n-k-1} u_{k-1}(b) / L_{n-k-1} u_{k}(b) .
$$

For (3.4) ensures that

$$
L_{k-1}\left(\alpha u_{k-1}+\beta u_{k}\right)(a) \neq 0, \quad L_{n-k-1}\left(\alpha u_{k-1}+\beta u_{k}\right)(b) \neq 0
$$


and $\alpha u_{k-1}+\beta u_{k}$ has exactly $k-1$ zeros at $a$ and exactly $n-k-1$ zeros at $b$. If $u(x, s)$ would have for every $s$ a zero near $a$ (or near $b$ ), in addition to its zeros at $a, s$, and $b$, then the limit solution $\lim _{s \rightarrow a^{+}} \bar{u}(x, s)=\alpha u_{k-1}+\beta u_{k}$ would have more zeros at $a$ (or at $b$ ) than is possible by (3.5). Thus, assuming (3.4), the first part of Proposition 3.3 is verified.

Now consider $s \rightarrow b^{-}$. By a similar argument, $\lim _{s \rightarrow b^{-}} \bar{u}(x, s)$ has at least $l-1$ zeros at $a$ and at least $(k-l)+(n-k-1)=n-l-1$ zeros at $b$. Hence we must have

$$
\lim _{s \rightarrow b^{-}} \bar{u}(x, s)=\gamma u_{l-1}+\delta u_{l} .
$$

In order to prove that for $s$ close to $b, u(x, s)$ has no zero in $(s, b)$, it suffices to show that no zero of $u(x, s)$ tends to $b$ when $s \rightarrow b^{-}$. This happens when

$$
L_{n-l-1}\left(\gamma u_{l-1}+\delta u_{l}\right)(b) \neq 0 \text {. }
$$

For, if $u(x, s)$ would have a zero near $b$, when $s \rightarrow b^{-}$, in addition to the zeros at $s$ and at $b$, then $\lim _{s \rightarrow b^{-}} \bar{u}(x, s)=\gamma u_{l-1}+\delta u_{l}$ would have more than $n-l-1$ zeros at $b$, in contradiction with (3.6).

When is (3.6) satisfied? By (3.2),

$$
\begin{aligned}
u(x, s)= & W\left(u_{l}, u_{l+1}, \ldots, u_{k-2}, \alpha u_{k-1}+\beta u_{k}\right)(s) u_{l-1}(x) \\
& +W\left(u_{l-1}, u_{l+1}, \ldots, u_{k-2}, \alpha u_{k-1}+\beta u_{k}\right)(s) u_{l}(x)+\cdots .
\end{aligned}
$$

So

$$
\gamma / \delta=\lim _{s \rightarrow b^{-}} \frac{W\left(u_{l}, \ldots, u_{k-2}, \alpha u_{k-1}+\beta u_{k}\right)}{W\left(u_{l-1}, u_{l+1}, \ldots, \alpha u_{k-1}+\beta u_{k}\right)(s)}
$$

and $\gamma / \delta$ is a bilinear function of $\alpha / \beta$. Therefore (3.6) holds for all but a single value of $\alpha / \beta$ :

$$
\alpha / \beta \neq \alpha_{3} / \beta_{3} \text {. }
$$

This completes the proof of Proposition 3.3.

We can summarize the situation as follows: For all values of $\alpha / \beta$ except the three which are specified in (3.4) and (3.7), $u(x, s)$ has for $s \rightarrow a^{+}$as many simple zeros in $(s, b)$ as $\alpha u_{k-1}+\beta u_{k}$ has in $(a, b)$; as $s$ increases, zeros of $u(x, s)$ leave $(s, b)$ one by one whenever $s$ crosses a zero of $\alpha z_{k-1}+\beta z_{k}$; and when $s \rightarrow b^{-}, u(x, s)$ has no zeros in $(s, b)$. Consequently, $\alpha z_{k-1}+\beta z_{k}$ and $\alpha u_{k-1}+\beta u_{k}$ have equal number of (simple) zeros in $(a, b)$.

To complete the proof of our theorem in the case $q=k$ it remains only to remove the restrictions (3.4), (3.7). Let $\alpha_{1} / \beta_{1}$ be one of the three exceptional values of $\alpha / \beta$, say $\alpha_{1} / \beta_{1}=-L_{n-k-1} u_{k-1}(b) / L_{n-k-1} u_{k}(b)$. Assuming $\beta_{1} \neq$ 0 ,

$$
\alpha u_{k-1}+\beta u_{k}=\beta / \beta_{1}\left(\alpha_{1} u_{k-1}+\beta_{1} u_{k}\right)+\beta\left(\alpha / \beta-\alpha_{1} / \beta_{1}\right) u_{k-1} .
$$

For $\alpha / \beta \neq \alpha_{1} / \beta_{1}, \alpha / \beta$ close to $\alpha_{1} / \beta_{1}, L_{n-k-1}\left(\alpha u_{k-1}+\beta u_{k}\right)(b) \neq 0$ and $\alpha u_{k-1}+\beta u_{k}$ has at $x=b$ a zero of exact multiplicity $n-k-1$. On the other hand $L_{n-k-1}\left(\alpha_{1} u_{k-1}+\beta_{1} u_{k}\right)(b)=0$ and $\alpha_{1} u_{k-1}+\beta_{1} u_{k}$ has at $x=b$ a zero 
of exact multiplicity $n-k$, for a zero of bigger multiplicity would contradict (2.3). Therefore,

$$
\begin{aligned}
\alpha u_{k-1}+\beta u_{k}= & \beta / \beta_{1}(x-b)^{n-k}[A+O(1)] \\
& +\beta\left(\alpha / \beta-\alpha_{1} / \beta_{1}\right)(x-b)^{n-k-1}[B+O(1)],
\end{aligned}
$$

where $A, B \neq 0$. It follows that for $\alpha / \beta$ close to $\alpha_{1} / \beta_{1}, \alpha u_{k-1}+\beta u_{k}$ has a simple zero close to $b$ and when $\alpha / \beta$ crosses $\alpha_{1} / \beta_{1}$, the simple zero of $\alpha u_{k-1}+\beta u_{k}$ passes from one side of $b$ to the other. The direction of this passage depends, of course, on the signs of the constants in (3.8).

Now, when $\alpha / \beta \neq \alpha_{1} / \beta_{1}$, it had been already proved that $\alpha u_{k-1}+\beta u_{k}$ and $\alpha z_{k-1}+\beta z_{k}$ have the same number of zeros in $(a, b)$. Therefore, when a zero of $\alpha u_{k-1}+\beta u_{k}$ passes through $b$ during the change of $\alpha / \beta$, a zero of $\alpha z_{k-1}+\beta z_{k}$ has to do the same. It follows that the number of the zeros of $\alpha u_{k-1}+\beta u_{k}$ and $\alpha z_{k-1}+\beta z_{k}$ is equal also for $\alpha / \beta=\alpha_{1} / \beta_{1}$. This completes the proof of Theorem 1.1 for $q=k$.

\section{Proof of Theorem 1.1 For $q \neq k$}

Recall the notation

$$
\begin{gathered}
z_{j}=W\left(u_{l-1}, \ldots, u_{k-2}, u_{j}\right), \quad j \notin\{l-1, \ldots, k-2\}, \\
\alpha z_{q-1}+\beta z_{q}=W\left(u_{l-1}, \ldots, u_{k-2}, \alpha u_{q-1}+\beta u_{q}\right) .
\end{gathered}
$$

Theorem 1.1 for $q>k$ will follow from the following

Proposition 4.1. The functions $\alpha z_{q-1}+\beta z_{q}$ and

$$
W_{M}\left(z_{k-1}, \ldots, z_{q-2}, \alpha z_{q-1}+\beta z_{q}\right)
$$

have an equal number of zeros, all simple, in $(a, b)$.

Suppose momentarily that Proposition 4.1 had been proved. By the discrete Wronskian identity [6, p. 61] applied to (4.1) and (4.2) we get

$$
\begin{aligned}
& W_{M}\left(z_{k-1}, \ldots, z_{q-2}, \alpha z_{q-1}+\beta z_{q}\right) \\
&= W_{L}\left(u_{l-1}, \ldots, u_{k-2}, u_{k-1}, \ldots, u_{q-2}, \alpha u_{q-1}+\beta u_{q}\right) \\
& \times W_{L}\left(\begin{array}{c}
u_{l-1}, \ldots, u_{k-2} \\
1, \ldots, k-l
\end{array}\right) \cdots W_{L}\left(\begin{array}{c}
u_{l-1}, \ldots, u_{k-2} \\
q-k, \ldots, q-l+1
\end{array}\right) .
\end{aligned}
$$

Since

$$
W\left(\begin{array}{c}
u_{l-1}, \ldots, u_{k-2} \\
i, \ldots, i+k-l+1
\end{array}\right) \neq 0 \text { in }(a, b),
$$

it follows from Proposition 4.1 that $\alpha z_{q-1}+\beta z_{q}$ and $W\left(u_{l-1}, \ldots, u_{q-2}, \alpha u_{q-1}\right.$ $\left.+\beta u_{q}\right)$ have an equal number of zeros in $(a, b)$. However, the last minor is exactly the one which is obtained when one chooses $\left\{u_{l-1}, \ldots, u_{q-2}\right\}$ as the pivotal block and so we encounter the situation which had been studied in the previous section! It follows by the result of the previous section that 
$W\left(u_{l-1}, \ldots, u_{q-2}, \alpha u_{q-1}+\beta u_{q}\right)$ has in $(a, b)$ as many zeros as $\alpha u_{q-1}+\beta u_{q}$ has, all of them simple. So Proposition 4.1 implies Theorem 1.1.

Thus, we have only to prove Proposition 4.1. Now, Proposition 4.1 reminds us of Theorem 1.1 with $q=k$, when the solutions $u_{l-1}, \ldots, u_{k-2}$, $\alpha u_{k-1}+\beta u_{k}$ of (1.1) are replaced by the solutions $z_{k-1}, \ldots, z_{q-2}, \alpha z_{q-1}+\beta z_{q}$ of (2.5). However, there is an essential difference: while $u_{l-1}, \ldots, u_{k}$ are defined as solutions of boundary value problems, $z_{k-1}, \ldots, z_{q}$ are defined by determinants. Nevertheless, Proposition 4.1 will be verified by repeating most of the arguments of the previous section literally. Difficulties arise only when we consider zeros of solutions $z_{j}$ near the endpoints $a$ and $b$. Since $a$ and $b$ are singular points for equation (2.6), the multiplicity of the zero of a function $M_{i} z$ is not necessarily equal to the number of quasiderivatives $M_{i} z, M_{i+1} z, \ldots$ which vanish there; and when zeros approach $x=a$ or $b$, we cannot simply sum up the number of vanishing quasiderivatives without a careful examination. Therefore we begin with a result about boundary values of minors at $a$ and $b$.

Lemma [2, Lemma 3.1]. If $l \leq k$ are of admissible parity, $0 \leq \alpha_{l-1}<\cdots<$ $\alpha_{k} \leq n-1$, then the function

$$
W\left(\begin{array}{c}
u_{l-1}, \ldots, u_{k} \\
\alpha_{l-1}, \ldots, \alpha_{k}
\end{array}\right)
$$

has exactly $\sum_{l-1}^{k}\left(i-\alpha_{i}\right)_{+}$zeros at $x=a$ and exactly $\sum_{l-1}^{k}\left(n-k+l+i-\alpha_{i}\right)_{+}$ zeros at $x=b$. If $r>k$ and $\alpha_{k}<\alpha_{r} \leq n-1$, then

$$
W\left(\begin{array}{c}
u_{l-1}, \ldots, u_{k}, u_{r} \\
\alpha_{l-1}, \ldots, \alpha_{k}, \alpha_{r}
\end{array}\right)
$$

has at least $\sum_{l-1}^{k}\left(i-\alpha_{i}\right)_{+}+\left(r-\alpha_{r}\right)_{+}$zeros at $x=a$ and at least $(n-\hat{r}-1-$ $\left.\alpha_{l-1}\right)+\sum_{l-1}^{k}\left(n-k+1+i-\alpha_{i}\right)_{+}+\left(n-l-1-\alpha_{r}\right)_{+}$zeros at $x=b$, where $\hat{r}$ equals $r$ or $r+1$ according to whether or not $r$ is of admissible parity.

After this preparation we turn to the series of arguments which will lead to the proof of Proposition 4.1. In (3.2) we replace $u_{l-1}, \ldots, u_{k-2}, \alpha u_{k-1}+\beta u_{k}$ by $z_{k-1}, \ldots, z_{q-2}, \alpha z_{q-1}+\beta z_{q}$ and define

$$
\begin{aligned}
& z(x, s)=\left|\begin{array}{rllc}
z_{k-1}(x), & \ldots, & z_{q-2}(x), & \alpha z_{q-1}+\beta z_{q}(x) \\
M_{0} z_{k-1}(s), & \ldots, & M_{0} z_{q-2}(s), & M_{0}\left(\alpha z_{q-1}+\beta z_{q}\right)(s) \\
\vdots & & & \vdots \\
M_{q-k-1} z_{k-1}(s), & \ldots, & & M_{q-k-1}\left(\alpha z_{q-1}+\beta z_{q}\right)(s)
\end{array}\right| \\
& =d_{k-1}(s) z_{k-1}+\cdots+d_{q-2}(s) z_{q-2}+d_{q-1}(s)\left(\alpha z_{q-1}+\beta z_{q}\right) .
\end{aligned}
$$


Here $d_{q-1}(s)=W_{M}\left(z_{k-1}, \ldots, z_{q-2}\right)(s)$ and by the discrete Wronskian identity

$$
\begin{aligned}
& d_{q-1}(s)= W_{L}\left(u_{l-1}, \ldots, u_{k-2}, u_{k-1}, \ldots, u_{q-2}\right)(s) \\
& \times W_{L}\left(\begin{array}{c}
u_{l-1}, \ldots, u_{k-2} \\
1, \ldots, k-l
\end{array}\right) \cdots W_{L}\left(\begin{array}{c}
u_{l-1}, \ldots, u_{k-2} \\
q-k-1, \ldots, q-l
\end{array}\right)(s) \neq 0, \\
& a<s<b .
\end{aligned}
$$

Proposition 4.2. One simple zero of $z(x, s)$ passes from $(s, b)$ to $(a, s)$ when and only when $s$ crosses a zero of $W_{M}\left(z_{k-1}, \ldots, z_{q-2}, \alpha z_{q-1}+\beta z_{q}\right)$ from left to right. This is the only way by which the number of the zeros of $z(x, s)$ in $(s, b)$ may change as $s$ varies in $(a, b)$.

The proof is parallel to that of Proposition 3.2. In order to show that no zero of $z(x, s)$ tends to $b$ as $s$ varies, we need the boundary values of $z(x, s)$ at $x=b$. This may be done as follows: By the Lemma, if $i+k-l<n$, the function

$$
M_{i} z_{r}=W\left(\begin{array}{c}
u_{l-1}, \ldots, u_{k-2}, u_{r} \\
i, \ldots, i+k-l
\end{array}\right) / W\left(\begin{array}{c}
u_{l-1}, \ldots, u_{k-2} \\
i+1, \ldots, i+k-l
\end{array}\right),
$$

has at $x=b$ a zero of multiplicity at least

$\left[(n-\hat{r}-1-i)_{+}+(n-k+1-i-1)_{+}(k-l)\right]-\left[(n-k+1-i-1)_{+}(k-l)\right]=(n-\hat{r}-1-i)_{+}$.

This implies, by the way, that the $M_{i} z_{r}, i=0, \ldots, n-k+l-1$, are continuous at $x=b$ in spite of the singularity of equation (2.5) at $x=b$. Now $z(x, s)=$ $d_{k-1} z_{k-1}+\cdots+d_{q-2} z_{q-2} d_{q-1}\left(\alpha z_{q-1}+\beta z_{q}\right)$ satisfies

$$
\begin{gathered}
M_{i} z(b)=0, \quad i=0, \ldots, n-q-2, \\
M_{n-q-1} z(b)=d_{q-1}(s)\left(\alpha M_{n-q-1} z_{q-1}+\beta M_{n-q-1} z_{q}\right)(b), \\
M_{n-q} z(b)=d_{q-1}(s)\left(\alpha M_{n-q} z_{q-1}+\beta M_{n-q} z_{q}\right)(b) .
\end{gathered}
$$

Since $d_{q-1}(s) \neq 0$ for $a<s<b$, the vanishing (or nonvanishing) of the functions $M_{n-q-1} z, M_{n-q} z$ at $b$ is independent of $s$. On the other hand, $M_{n-q-1} z(x, s), M_{n-q} z(x, s)$ are continuous near $x=b$ for $a<s<b$ and if a zero of $z(x, s)$ would tend to $b$ as $s$ varies, an additional quasiderivative would vanish at $x=b$, in contradiction to the previous remark. Consequently, no zero of $z(x, s)$ may tend to $b$ when $s$ varies in $(a, b)$.

Proposition 4.3. When $s$ is sufficiently close to $a, z(x, s)$ has in $(s, b)$ exactly as many zeros as $\alpha z_{q-1}+\beta z_{q}$ has in $(a, b)$. When $s$ is sufficiently close to $b$, $z(x, s)$ has no zeros in $(s, b)$.

We normalize $z(x, s)$ and define

$$
\bar{z}(x, s)=\left[d_{k-1}(s) z_{k-1}+\cdots+d_{q-2}(s) z_{q-2}+d_{q-1}(s)\left(\alpha z_{q-1}+\beta z_{q}\right)\right] / \sum_{k-1}^{q-1}\left|d_{i}(s)\right| .
$$

By the normalization, $\bar{z}(x, s)$ (or a subsequence) converges uniformly on any compact interval to a nontrivial solution. In order to understand what happens 
when $s \rightarrow a^{+}$, we need to know about the behavior of

$$
M_{i} z_{r}=W\left(\begin{array}{c}
u_{l-1}, \ldots, u_{k-2}, u_{r} \\
i, \ldots, i+k-l
\end{array}\right) / W\left(\begin{array}{c}
u_{l-1}, \ldots, u_{k-2} \\
i+1, \ldots, i+k-l
\end{array}\right)
$$

near $x=a$. If $i+k-l<n$, then, by the Lemma, $M_{i} z_{r}$ has at $x=a$ a zero of multiplicity at least $\left[(l-1-i)_{+}(k-l)+(r-i-k+l)_{+}\right]-\left[(l-1-i-1)_{+}(k-l)\right]$. If $i \leq l-2$, the multiplicity of the zero of $M_{i} z_{r}$ at $x=a$ is at least $r-i$ and if $l-1 \leq i \leq n-k+l$, the multiplicity is at least $[r-(i+k-l)]_{+}$. Anyway, $M_{i} z_{r}$ is continuous near $x=a$ at least for $i=0, \ldots, n-k+l$. (Note the difference between the multiplicity of the zero of $M_{l-2} z_{r}$ and that of $M_{l-1} z_{r}=\sigma_{l-1}\left(M_{l-2} z_{r}\right)^{\prime}$. This is due to the singularity of $\sigma_{l-1}$ at $\left.a\right)$. For $i=r-k+l$ we expand the numerator of $M_{r-k+l} z_{r}(a)$ according to its last column and get

$$
W\left(\begin{array}{c}
u_{l-1}, \ldots, u_{k-2}, u_{r} \\
r-k+l, \ldots, r
\end{array}\right)(a)=W\left(\begin{array}{c}
u_{l-1}, \ldots, u_{k-2} \\
r-k+l, \ldots, r-1
\end{array}\right)(a) L_{r} u_{r}(a) \neq 0 .
$$

Therefore

$$
\begin{aligned}
& M_{i} z_{r}(a)=0, \quad i=0, \ldots, r-k+l-1, \\
& M_{r-k+l} z_{r}(a) \neq 0 .
\end{aligned}
$$

Since $\bar{z}(x, s)$ is a combination of $z_{k-1}, \ldots, z_{q}$,

$$
M_{i} \bar{z}(a)=0, \quad i=0, \ldots, l-2 .
$$

Now $\bar{z}(x, s)$ also has $q-k$ zeros at $x=s$, therefore by Rolle's theorem each of the functions $M_{l-1} \bar{z}(x, s), \ldots, M_{l-1+q-k-1} \bar{z}(x, s)$ has one or more zeros in $(a, s)$. As $\bar{z}(x, s)$ (or a subsequence) converges uniformly when $s \rightarrow a^{+}$, the limit solution and its quasiderivatives of orders $0, \ldots, q-k+$ $l-2$ vanish at $a$. On the other hand, $\lim _{s \rightarrow a^{+}} \bar{z}(x, s)$ is a combination of $z_{k-1}, \ldots, z_{q-2}, \alpha z_{q-1}+\beta z_{q}$, so by (4.3) it may have $q-k+l-1$ vanishing quasiderivatives at $x=a$ if and only if, up to a constant factor,

$$
\lim _{s \rightarrow a^{+}} \bar{z}(x, s)=\alpha z_{q-1}+\beta z_{q} .
$$

In order to prove that for $s$ sufficiently close to $a, z(x, s)$ and $\alpha z_{q-1}+\beta z_{q}$ have an equal number of zeros in $(s, b)$, it suffices to show that no zero of $z(x, s)$, except $s$, tends to the endpoints $a$ or $b$ when $s \rightarrow a^{+}$. This is definitely the case if

$$
\alpha / \beta \neq 0, \quad \alpha / \beta \neq-M_{n-q-1} z_{q-1}(b) / M_{n-q-1} z_{q}(b) .
$$

For (4.4) ensures that

$$
M_{q-k+l-1}\left(\alpha z_{q-1}+\beta z_{q}\right)(a) \neq 0, \quad M_{n-q-1}\left(\alpha z_{q-1}+\beta z_{q}\right)(b) \neq 0 .
$$

But if $z(x, s)$ would have an additional zero near $a$ (or near $b$ ) for every $s$, then the limit solution $\lim _{s \rightarrow a^{+}} \bar{z}(x, s)=\alpha z_{q-1}+\beta z_{q}$ would violate (4.5).

Thus, we have proved the first half of Proposition 4.3. The other half, concerning $s \rightarrow b^{-}$, is proved by a similar modification of the corresponding part of the proof of Proposition 3.3 and it will be omitted. 
The singular values of $\alpha / \beta$ are treated as in $\S 3$. This completes the proof of Theorems 1.1 and 1.2 for every $q \geq k$.

For the case $q \leq l-2$, Theorem 1.1 is proved by similar arguments. Proposition 3.3, for example, will be replaced by the following: When $s$ is sufficiently close to $b, u(x, s)$ has in $(a, s)$ exactly as many zeros as $\alpha u_{l-3}+\beta u_{l-2}$ has in $(a, b)$. When $s$ is sufficiently close to $a, u(x, s)$ may have in $(a, s)$ at most one zero.

In the analogue of Proposition 4.1, the zeros of $\alpha z_{q-1}+\beta z_{q}$ will be compared with those of $W_{M}\left(\alpha z_{q-1}+\beta z_{q}, z_{q+1}, \ldots, z_{l-2}\right)$. The differences between the cases $q \geq k$ and $q \leq l-2$ are due to the asymmetry between the boundary conditions (2.2) at $x=a$ and at $x=b$.

\section{SOME APPLICATIONS}

The number of zeros of $\alpha u_{q-1}+\beta u_{q}$ is closely related to oscillatory properties of equation (1.1). Recall that the $(q, n-q)$-type conjugate point of $x=a$ is the smallest value of $c, c>a$, such that the boundary value problem

$$
\begin{aligned}
& L_{i} y(a)=0, \quad i=0, \ldots, k-1, \\
& L_{j} y(c)=0, \quad j=0, \ldots, n-k-1,
\end{aligned}
$$

has a nontrivial solution. More generally, the $i$ th $(k, n-k)$-type conjugate point of $a, i \geq 1$, is the $i$ th value of $c$ in $(a, \infty)$ such that $(5.1)$ has a nontrivial solution. It is known that the number of zeros of $u_{q}$ in $(a, b)$ is equal to the number of $(q, n-q)$-type conjugate points of $x=a$ which are in $(a, b)$. Hence,

Corollary. The number of the zeros of $z_{q}=W\left(u_{l-1}, \ldots, u_{k}, u_{q}\right)$ in $(a, b)$ is equal to the number of the $(q, n-q)$-type conjugate points of a for equation (1.1) which are in $(a, b)$. For every other combination $\alpha u_{q-1}+\beta u_{q}$, the number of zeros of $W\left(u_{l-1}, \ldots, u_{k}, \alpha u_{q-1}+\beta u_{q}\right)$ may differ from the number mentioned above by at most 1 .

Indeed, for two independent solutions $\alpha u_{q-1}+\beta u_{q}$ and $\gamma u_{q-1}+\delta u_{q}$, $W\left(\alpha u_{q-1}+\beta u_{q}, \gamma u_{q-1}+\delta u_{q}\right)=(\alpha \delta-\beta \gamma) W\left(u_{q-1}, u_{q}\right) \neq 0$ on $(a, b)$, so their zeros separate each other in $(a, b)$ and their number may differ by at most 1 . Hence, the same holds for the number of zeros of the minors $W\left(u_{l-1}, \ldots, u_{k}\right.$, $\left.u_{q}\right)$ and $W\left(u_{l-1}, \ldots, u_{k}, \alpha u_{q-1}+\beta u_{q}\right)$.

Up to now, we have discussed equation (1.1) only on a finite interval $(a, b)$. Let us consider it now on $(a, \infty)$. First we point out the dependence of the solutions $u_{i}$ of $(2.2)_{i}, i=0, \ldots, n-1$, on $b$ and denote them from now on by $u_{i}(x, b)$. Next, we let $b \rightarrow \infty$ and define

$$
\tilde{u}_{i}(x) \stackrel{\text { def }}{=} \lim _{b \rightarrow \infty} u_{i}(x, b),
$$

when $b \rightarrow \infty$ along a suitable sequence of values. $\left\{\tilde{u}_{0}(x), \ldots, \tilde{u}_{n-1}(x)\right\}$ which 
are defined so, are a basis [1], and they satisfy Proposition $2.2[2,4]$. In particular,

$$
S\left(\alpha \tilde{u}_{q-1}+\beta \tilde{u}_{q}, x\right) \equiv q, \quad a<x<\infty,
$$

and all zeros of $\alpha \tilde{u}_{q-1}+\beta \tilde{u}_{q}$ on $(a, \infty)$ are simple. Moreover, $\tilde{u}_{0}, \ldots, \tilde{u}_{n-1}$ also satisfy Proposition 2.3 for $b=\infty$ [3, Theorem 2.5].

Recall that by Proposition 2.1, $S\left(y, x^{+}\right)$is an integer valued, nondecreasing function of $x$ and the values of $S\left(y, x^{+}\right)$are integers of admissible parities. Accordingly, we defined $S_{k}$ in [1] as the set of solutions of $(1.1)$ on $(a, \infty)$ such that $S\left(y, x^{+}\right) \equiv k$ on some ray $\left(x_{0}, \infty\right)$. The partition of the solution space into the sets $S_{k}$ for admissible values of $k$ is discussed in $[1,5]$. One property of $S_{k}$ that we are going to use here is that the elements of $S_{k}$ are either all oscillatory or all nonoscillatory solutions of (1.1).

Now we consider the sets $S_{q}$ for equations (1.1) and (2.6) and denote them by $S_{q}(L)$ and $S_{q}(M)$, respectively. By inequalities (2.3) and (2.7) applied to $(a, \infty)$, we get that

$$
\alpha \tilde{u}_{q-1}+\beta \tilde{u}_{q} \in S_{q}(L) \text { and } \alpha \tilde{z}_{q-1}+\beta \tilde{z}_{q} \in S_{q}(M) .
$$

But when we let $b \rightarrow \infty$, we obtain from our present results that $\tilde{u}_{q}$ and $\tilde{z}_{q}$ have on $(a, \infty)$ an equal number, finite or infinite, of simple zeros. Hence

Corollary. The solutions of $(1.1)$ in $S_{q}(L)$ are oscillatory if and only if the solutions of (2.6) in $S_{q}(M)$ are oscillatory.

Equation (1.1) is $(q, n-q)$-disconjugate on $(a, \infty)$ if and only if equation (2.6) is $(q, n-q)$-disconjugate on $(a, \infty)$.

\section{REFERENCES}

1. U. Elias, $A$ classification of solutions of a differential equation according to their asymptotic behaviour, Proc. Roy. Soc. Edinburgh Sect. A 83 (1979), 25-38.

2. Minors of the wronskian of the differential equation $L_{n} y+p(x)=0$, Proc. Roy. Soc. Edinburgh Sect. A 106 (1987), 341-359.

3. _- Minors of the wronskian of the differential equation $L_{n} y+p(x)=0$. II: Dominance of solutions, Proc. Roy. Soc. Edinburgh Sect. A 108 (1988), 229-239.

4. G. J. Etgen, G. D. Jones, and W. E. Taylor, On the factorization of ordinary linear differential operators, Trans. Amer. Math. Soc. 297 (1986), 717-728.

5. G. D. Jones, Growth properties of solutions of a linear differential equation, Proc. Roy. Soc. Edinburgh Sect. A 104 (1986), 127-135.

6. S. Karlin, Total positivity, Stanford Univ. Press, Stanford, Calif., 1968.

7. Z. Nehari, Disconjugate differential operators, Trans. Amer. Math. Soc. 129 (1967), 500516.

Department of Mathematics, Technion-Israel Institute of Technology, Haifa 32000, ISRAEL 